\title{
Diferenças de gênero e valores relativos ao trabalho ${ }^{1}$
}

\author{
Margareth Ribeiro Machado Santos e Silva ${ }^{2}$ \\ Faculdade Estácio de Sá de Goiás, Goiânia-GO, Brasil \\ Helenides Mendonça \\ Daniela Sacramento Zanini \\ Pontifícia Universidade Católica de Goiás, Goiânia-GO, Brasil
}

\begin{abstract}
Resumo: Este estudo investiga as diferenças no estabelecimento das prioridades axiológicas laborais e a satisfação das metas motivacionais relacionadas ao trabalho em relação ao gênero. Participaram 178 universitários de diversos cursos que responderam os instrumentos de forma coletiva e presencial em sala de aula. Como medida utilizou-se duas escalas de valores relativos ao trabalho: uma sobre prioridade atribuída a metas motivacionais (alphas entre 0,81 e 0,88 ) e outra sobre a satisfação de tais metas (alphas entre 0,86 e 0,95). Os dados analisados, utilizando-se da técnica estatística ANOVA, demonstraram que as mulheres priorizam valores laborais de autonomia intelectual e criatividade; e priorizam, mais que os homens, segurança e ordem na vida. Não foram identificadas diferenças significativas entre gênero e satisfação dos valores laborais. Esses resultados são discutidos ressaltando-se a idéia de uma caracterização específica de gênero no mundo do trabalho, possibilitando a convivência com princípios e metas motivacionais paradoxais.
\end{abstract}

Palavras-chave: valores, gênero, trabalho.

\section{Differences of gender and work-related values}

\begin{abstract}
This study investigates the differences in establishing axiological work priorities and satisfaction with work-related goals in relation to gender. The participants were 178 undergraduate students from different programs who filled out the instruments in classroom. Two scales of work-related values were used: one about the level of priority given to work-related motivational goals (alphas between 0.81 and 0.88 ), and the other about the level of satisfaction of such goals (alphas between 0.86 and 0.95 ). Data analyzed through the statistic technique ANOVA revealed that women give priority to work values such as intellectual autonomy and creativity, and more than men, they give priority to security and order in life. Significant differences were not found between gender and satisfaction of work values. These results are discussed highlighting the idea of a specific characterization of gender in the world of work, which enables the co-existence of paradoxical principles and motivational goals.
\end{abstract}

Keywords: values, gender, labor.

\section{Diferencias de género y valores relativos al trabajo}

\begin{abstract}
Resumen: Este estudio investiga las diferencias entre los valores de trabajo y la satisfacción de las metas motivacionales relacionadas al trabajo en relación al género. Participaron 178 universitarios de diversas carreras que contestaron los instrumentos de forma colectiva y presencial en su aula. Como medida se utilizó dos escalas de valores relativos al trabajo: una sobre la prioridad atribuida a metas motivacionales y otra que investiga la satisfacción de estas metas (alphas entre 0,86 y 0,95). Los datos, analizados utilizando la técnica estadística ANOVA, demostraron que las mujeres priorizan valores laborales de autonomía intelectual y creatividad, y priorizan, más que los hombres, la seguridad y el orden en la vida. No fueran identificadas diferencias significativas entre género y la satisfacción de los valores laborales. Estos resultados son discutidos resaltándose la idea de una caracterización específica de género en el mundo del trabajo, posibilitando la convivencia con principios y metas motivacionales paradojales.
\end{abstract}

Palabras clave: valores, gênero, trabajo.

Nas últimas décadas, mudanças significativas no perfil feminino evidenciaram conquistas de posições no trabalho que, tradicionalmente, costumavam ser masculinas (Abramo, 2002; Torres, 2006). Segundo o Instituto Brasileiro de Geografia e Estatística [IBGE] (2006), o percentual de

1 Apoio: CAPES. Este artigo é derivado da Dissertação de Mestrado defendida pela primeira autora, sob a orientação da segunda, junto ao Programa de Pós-graduação Stricto Sensu em Psicologia da Pontifícia Universidade Católica de Góias, Goiânia, SP.

2 Endereço para correspondência:

Helenides Mendonça. Rua SB, 14, Qd. 19, Lt. 08, Portal do Sol I. CEP. 74.884-600. Goiânia-GO.E-mail: helenides@uol.com.br mulheres no conjunto de trabalhadores brasileiros cresceu de $21 \%$, em 1970, para 43,5\%, em 2005. O aumento quantitativamente significativo de participação feminina no mercado de trabalho e a mudança de seu modelo laboral não permitem mais considerar a mulher "uma força de trabalho secundária" (Fischer, 2001; Venturi \& Recamán, 2005).

Esses indicadores sugerem progressiva conquista de igualdade entre os gêneros no mercado de trabalho, entretanto, o cenário ainda se configura com poucas oportunidades de acesso feminino a novas profissões, a ocupações que envolvam novas tecnologias, a postos de trabalho mais bem remunerados ou de maior responsabilidade hierárquica (Abramo, 2002). 
Pesquisas recentes parecem confirmar essas postulações. Venturi e Recamán (2005), por exemplo, ao estudarem os papéis desempenhados por homens e mulheres na divisão do trabalho (remunerado ou não) entre casais brasileiros, encontraram que $93 \%$ dos homens e $45 \%$ das mulheres são provedores. Contudo, entre as mulheres provedoras, $97 \%$ ainda executam ou chefiam atividades domésticas, contra apenas $20 \%$ dos homens provedores. Esse grau de desigualdade entre gênero na divisão do trabalho doméstico evidencia a existência e o peso da dupla jornada de trabalho remunerado e não remunerado, sobre a mulher.

No mercado de trabalho, dada à condição de mulher, historicamente referenciada como o "sexo frágil", débil ou inferior em relação ao homem (Fischer, 2001; Fiúza, 2001), a força de trabalho feminina é vendida a um valor inferior em relação ao trabalho masculino. O trabalho feminino, apesar de ser representativo para o orçamento familiar, concentra as atividades em setores extensivos ao âmbito doméstico, a exemplo da educação, saúde, assistência social, enfermagem e têxtil, em que se desenvolvem tarefas que o homem, muitas vezes, nega-se a fazer e permanece distante das esferas de comando e decisão (Venturini \& Recaman, 2005).

Estudos desenvolvidos por Beutel e Marini (1995) demonstram que as diferenças de gênero em diversos papéis sociais podem ser produzidas pela adesão a determinados valores sociais. Partindo do pressuposto de que os valores são metas motivacionais que guiam a vida dos indivíduos, das organizações e da sociedade como um todo (Tamayo, 1994; Tamayo \& Schwartz, 1993), a atribuição de maior importância a valores diferentes feita por homens e mulheres pode promover características específicas no desempenho do papel profissional. Esses aspectos, somados a outros que estão relacionados às condições do ambiente laboral, podem contribuir decisivamente para as desigualdades de gênero no trabalho. Portanto, integrar os valores ao estudo das diferenças sexuais, pode contribuir para a compreensão do sexismo, entendido como "um conjunto de estereotipos sobre a avaliação cognitiva, afetiva e atitudinal acerca do papel apropriado na sociedade dirigida aos indivíduos de acordo com o sexo" (Formiga, 2007, p.383), e de outras "barreiras invisíveis" que dificultam a igualdade de gênero no mundo do trabalho.

Considerando tais premissas, este artigo investiga se homens e mulheres estabelecem diferentes prioridades axiológicas laborais, bem como se elas são igualmente realizadas por ambos no trabalho.

\section{O conceito de gênero e suas implicações}

Segundo Scott (1995, p. 75), o termo gênero é utilizado para apresentar "o discurso da diferença dos sexos". Trata-se de "uma forma de se referir às origens exclusivamente sociais das identidades subjetivas de homens e de mulheres", o que exclui, portanto, explicações biológicas. A mesma autora considera que originalmente o termo tem uma conotação neutra. Embora seu uso recente direcione para que seja compreendido como sinônimo de "mulheres", em razão de ser esse, em comparação com os homens, o grupo minoritário, pelas condições históricas impostas a essa categoria de análise. A autora sugere que "qualquer informação sobre mulheres é necessariamente informação sobre os homens, pois o estudo de um implica o estudo do outro" (Scott, 1995, p. 75).

Assim, gênero feminino e gênero masculino são categorias relacionais, por isso implica na construção de indicadores com objetivo de revelar a participação dos dois sexos (Scott, 1995). Nota-se, então, que os estudos de gênero partem de comparações feitas ao grupo social de referência, sendo que para o grupo de mulheres o referente é o grupo de homens e vice-versa. Nessa perspectiva pode-se dizer que a idéia de feminino apenas existe a partir do reconhecimento da diferença do masculino, assim como a idéia do masculino só existe a partir do reconhecimento da diferença do feminino.

As diferenças entre sexos têm sido comumente consideradas por meio de várias abordagens teóricas. A psicologia evolutiva considera que mecanismos psicológicos de diferenciação sexual são desenvolvidos evolutivamente, ao longo do tempo, em decorrência de adaptação a problemas impostos por diferentes pressões sobre homens e mulheres. Tais processos de natureza afetiva e cognitiva podem ser diferentes em virtude de contextos distintos, providos por diferentes culturas (Schwartz, 2005).

Ferreira e Assmar (2005) postulam que as desigualdades são socialmente apreendidas e continuamente reforçadas pelo consenso social, motivo pelo qual a modificação delas se torna difícil.

Por outro lado, outros autores tendem a atribuir as diferenças de gênero à divisão social do trabalho, originada pelas diferenças físicas entre homem e mulher, em interação com condições ecológicas e sociais. Valendo-se dessa concepção, os diferentes papéis sociais adotados por homens e mulheres seriam o resultado de um processo de construção social que, em última instância, revelam também uma acomodação social a diferentes restrições e oportunidades biológicas relacionadas ao sexo do indivíduo (Schwartz, 2005). Assim, podemos dizer que a contribuição desta abordagem reside na consideração de que o contexto societal ou cultural influi sobre as diferenças de gênero.

Scott (1995), avaliando as influências das teorias feministas sobre a percepção das diferenças entre gênero, relata que essas associam as diferenças de gênero à cultura patriarcal, que teria destinado o espaço público ao homem e o privado à mulher, desenvolvendo representações masculinas associadas à dominação e representações femininas relacionadas à submissão e à responsabilidade pelo lar. Além disso, as teorias feministas de orientações marxistas ou psicanalíticas ainda relacionam, respectivamente, tais diferenças a modos de produção e estruturas econômico-sociais ou à influência de processos de criação da identidade: experiência concreta com os pais e influência da linguagem na interpretação e representação do gênero (Scott, 1995).

Gênero é, portanto, uma categoria estreitamente relacionada à divisão sexual do trabalho e, consequentemente, aos novos determinantes do mundo do trabalho (Torres, 2006), motivo pelo qual tem sido incluído em estudos de diversas 
áreas do conhecimento. De acordo com Ferreira e Assmar (2005), em Psicologia, especialmente na área do trabalho, diversas pesquisas identificam que as organizações não reconhecem as mulheres trabalhadoras como possuidoras das qualidades necessárias ao sucesso gerencial.

Para Beutel e Marini (1995), os valores podem produzir diferenças de gênero nos diferentes papéis sociais e, dessa forma, constituir uma categoria de análise importante para a compreensão das diferenças de gênero no mundo do traba1ho. Esses autores demonstraram que as mulheres se envolvem, mais que os homens com atividades cuidadoras e que também dispõem de mais apoio socioemocional. Este estudo é de especial relevância para essa área, na medida em que essa constatação promoverá a postulação de mecanismos de identificação e possibilidades de intervenção para reduzir o sexismo nas organizações.

\section{Valores gerais e valores relativos ao trabalho}

Os valores em geral e relativos ao trabalho são construídos pelas pessoas de acordo com sua cultura, sociedade e personalidade, e influenciam suas atitudes e seus comportamentos (Belo, Gouveia, \& Raymundo, 2005). Funcionam como padrões de conduta, ao posicionarem as pessoas diante de princípios sociais (Rokeach, 1973). Pode-se dizer que os valores compreendem uma crença; pertencem a fins desejáveis; transcendem situações específicas; guiam a seleção ou avaliação de comportamentos; podem ser ordenados ou hierarquizados formando um sistema de prioridades axiológicas. Sua análise pode ser considerada no nível de uma estrutura geral mais ampla e abstrata ou no âmbito de contextos específicos como, por exemplo, a família, o trabalho ou o comportamento (Palermo \& Evans, 2007).

Baseado no modelo teórico elaborado por Rokeach (1973) e em estudos transculturais, Schwartz (2005) criou a Teoria dos Valores Básicos, que identifica dez tipos motivacionais ou grupamento de valores similares, agrupados a seguir de acordo com o tipo motivacional (e os valores que representa): 1) hedonismo: metas de prazer e gratificação para si mesmo (prazer, vida estimulante e auto-indulgência); 2) realização: sucesso pessoal obtido pela competência, visando o reconhecimento social (sucesso, capacidade, ambição e influência); 3) poder: busca e preservação de status, prestígio e controle sobre pessoas e recursos (poder social, autoridade, riqueza, vaidade); 4) autodeterminação: independência de pensamento e escolha das ações (criatividade, liberdade, independência, curiosidade, autodeterminação, autorrespeito e privacidade); 5) conformidade: busca de contenção dos impulsos de transgressão das normas sociais (polidez, autodisciplina e obediência); 6) benevolência: busca de preservação e da promoção de pessoas com as quais mantém contato (utilidade, honestidade, trabalho, sentido da vida, lealdade, responsabilidade e indulgência); 7) segurança: tem como meta a defesa da harmonia e da estabilidade da sociedade, das relações e do self (segurança da família, segurança nacional, senso de pertencer, ordem social, saúde e limpeza); 8) tradição: sentimento de respeito, de comprometimento e de aceitação de costumes e ideias da cultura e da religião (moderação, humildade, devoção, respeito pela tradição); 9) estimulação: busca de excitação, novidades e desafios na vida (audácia, vida excitante, vida variada); 10) universalismo: busca do entendimento, da compreensão, da tolerância, da apreciação e da proteção do bem-estar de todas as pessoas e da natureza (sabedoria, justiça social, igualdade, um mundo de paz, um mundo de beleza, união com a natureza, proteção do meio ambiente e harmonia).

Os Tipos Motivacionais são estruturados num continuum de motivações e se organizam hierarquicamente em virtude da importância relativa e de suas conseqüências práticas, psicológicas e sociais. A estrutura reflete compatibilidades entre os valores adjacentes e conflitos entre os tipos situados em lados opostos. Segundo Ros, Schwartz e Surkiss (1999), podem ser agrupados entre si, formando quatro fatores de ordem superior, que representam duas dimensões bipolares (Figura 1).

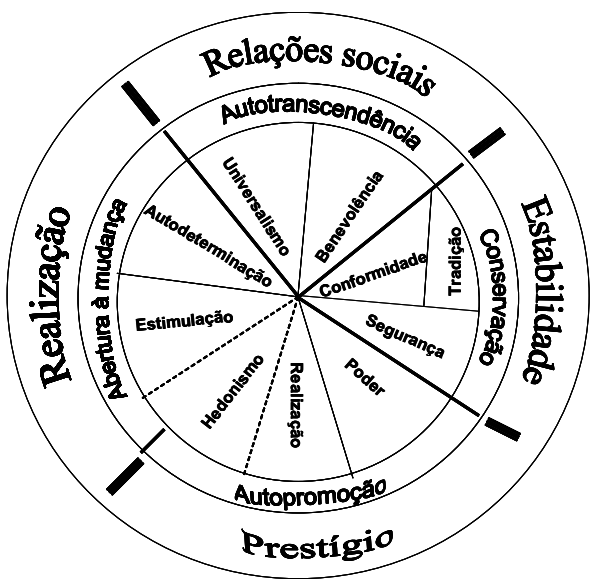

Figura 1. Estrutura motivacional dos valores humanos e fatores de ordem superior. Adaptada de Schwartz (1992) e Ros e cols., 1999

Em um dos eixos, observa-se a dimensão "autotranscendência x autopromoção" baseada na motivação do indivíduo para promover o bem-estar dos outros e da natureza, em oposição à motivação para promover seu bem-estar. No outro eixo, nota-se a dimensão "abertura à mudança x conservação", baseada na motivação do indivíduo para seguir seus próprios interesses intelectuais e afetivos, através de caminhos incertos, versus a motivação para preservar o status quo e as práticas tradicionais (Tamayo \& Schwartz, 1993).

Para Schwartz (2005, p. 22), "os indivíduos têm prioridades ou hierarquias axiológicas diferentes". Algumas pesquisas têm dado suporte a esta afirmação no que refere à diferença de gênero (Sagie, Elizur, \& Koslowsky, 1996; Jensen, White, \& Singh, 1990; Manhardt, 1972), ao passo que outras apresentam resultados contrários (Mottaz, 1986; Cheung \& Scherling, 1999). Porto (2004) considera que esses resultados são de difícil comparação, porque decorrem 
do uso de medidas e metodologias diferentes, de amostras restritas a determinada região, além de utilizarem valores únicos e não uma estrutura de valores. Schwartz e Rubel (2005) também consideram que alguns estudos não se reportam à importância dos valores ou priorização, e sim ao significado deles para homens e mulheres.

Em um estudo em que objetivavam identificar diferenças de gênero na priorização axiológica, Schwartz e Rubel (2005) realizaram pesquisa envolvendo 77.528 participantes de 70 países. Encontraram diferenças significativas entre os valores relativos à força, estimulação, ao hedonismo e à realização, priorizados pelos homens, e à benevolência e ao universalismo, priorizados pelas mulheres.

A partir desse estudo, os mesmos autores concluíram que entre estudantes universitários, distintamente de amostras gerais, as diferenças de gênero são pequenas e variam conforme a cultura. Reportaram-se, ainda, à teoria evolucionista, citada anteriormente, para explicar, por exemplo, a priorização feminina do valor benevolência, que estaria relacionada à necessidade de se adaptar às condições imprescindíveis à criação dos filhos, bem como a maior importância atribuída pelos homens à estimulação e realização, que seriam concernentes à competitividade deles para assegurar seu status social. Já a teoria social dos papéis permite associar a priorização feminina do valor segurança à sua maior vulnerabilidade social e status (Scott, 1995). No Brasil, Tamayo (1994) identificou que mulheres adolescentes priorizam mais o tipo motivacional autodeterminação.

Embora as conclusões referentes à priorização de valores gerais por homens e mulheres possam ser, hipoteticamente, consideradas para os valores relativos ao trabalho, têm sido realizados estudos, direcionados especificamente, para os valores laborais. Para cada aspecto da vida - como, por exemplo, o trabalho - as pessoas apresentam estrutura de valores específica, relacionada com a estrutura geral (Schwartz, 1992). Isso se dá em razão de que o contexto do trabalho implica a existência de variáveis e dinâmicas próprias, que podem influenciar significativamente o desenvolvimento de valores que lhe são específicos.

Os valores relativos ao trabalho têm recebido diversas definições. Neste estudo, será considerada a definição proposta por Ros e cols. (1999, p. 54) de que são "princípios ou crenças sobre comportamentos ou metas desejáveis, hierarquicamente organizados, que guiam as avaliações sobre os resultados e contexto do trabalho, bem como o seu comportamento no trabalho e a escolha de alternativas de trabalho".

Os valores laborais estão relacionados às dimensões de segunda ordem da estrutura proposta por Ros e cols. (1999) e podem ser de quatro tipos, conforme especificados a seguir: intrínsecos (realização) - quando se referem a metas obtidas pelo conteúdo do próprio trabalho, associados à abertura à mudança; extrínsecos ou materiais (estabilidade) - referentes a metas obtidas pelo resultado do trabalho, relacionados ao conservadorismo, pela busca de segurança e manutenção do status quo; sociais ou afetivos - quando se referem a metas relacionais, enfatizam o bem-estar da sociedade e estão associados à autotranscendência; e prestígio - em que há busca de prestígio, poder e sucesso pessoal, por meio do trabalho, relacionado à autopromoção.

Os valores relativos ao trabalho envolvem aspectos cognitivos, motivacionais e hierárquicos, assim como os valores gerais. Segundo Porto (2004), o desenvolvimento desses valores pode ser influenciado por mudanças de nível socioeconômico, pela origem social com mediação das experiências acadêmicas, e de trabalho, pela qualidade da experiência do trabalho. Ros e cols. (1999) identificaram em pesquisa com universitários, que a escolha da profissão exerceu maior influência sobre a priorização dos valores que a socialização no trabalho.

Porto e Tamayo (2003) desenvolveram e validaram a Escala de Valores Relativos ao Trabalho - EVT, que, após ter sido respondida por 394 pessoas, obteve bom índice de confiabilidade, o que a recomenda para pesquisa e diagnóstico. Essa escala considera os quatro tipos de valores laborais propostos por Ros e cols. (1999) - realização no trabalho ou intrínseco, relações sociais, prestígio e estabilidade ou extrínseco - e os relacionam, respectivamente, com os fatores de segunda ordem de Schwartz - abertura à mudança, autotranscendência, autopromoção e conservação (Porto, 2004).

Utilizando a EVT em pesquisa com 1.083 estudantes universitários brasileiros com idade média de 21 anos (DP = 4,12 ), Porto (2004), contrariamente ao encontrado em outras pesquisas em geral, identificou que as mulheres valorizam mais a busca de prazer e realização pessoal e profissional que as relações sociais. Como possibilidade explicativa, considera o fato de estudantes universitários questionarem fortemente os papéis de gênero. Argumenta ainda que mulheres, no ambiente de trabalho, poderiam buscar metas diferentes das desejadas em outros contextos de vida, caracterizando um mecanismo compensatório. Contudo, não foram identificados outros estudos sobre valores e gêneros utilizando a EVT a fim de possibilitar comparação de dados e comprovação da hipótese explicativa discutida por Porto (2004).

Em vista disso, este artigo busca identificar a priorização axiológica de homens e mulheres, estudantes universitários, por meio da escala EVT.

\section{Método}

\section{Participantes}

A amostra foi selecionada seguindo o critério de conveniência. Participaram do estudo 178 estudantes de diversos cursos universitários de uma universidade privada. Os participantes apresentavam idade média de 30,46 anos (DP = 8,2 ), sendo $59,6 \%$ do sexo feminino. Os participantes apresentavam tempo médio de serviço equivalente a 5 anos, e $41 \%$ desempenhavam funções de gestão.

\section{Instrumento}

Foi utilizada a EVT, na qual 45 valores deviam ser avaliados pelos indivíduos, segundo suas prioridades axiológicas, 
em uma escala de cinco pontos, variando de "nada importante" (1) a "muito importante" (5). Esses valores se agrupam em quatro fatores com valores alphas de 0,88 para Realização; 0,88 para Relações Sociais; 0,87 para Prestigio; e 0,81 para Estabilidade (Porto \& Tamayo, 2003).

Além disso, a escala original de EVT foi adaptada, passando a contar com uma segunda parte, na qual o respondente registrava se a organização onde trabalhava lhe permitia satisfazer seus valores relativos ao trabalho, na mesma escala de cinco pontos, variando de "não possibilita" (1) a "possibilita extremamente" (5). Tal adaptação permitiu identificar a realização ou não realização dos valores relativos ao trabalho, no ambiente laboral.

Os fatores considerados na escala adaptada, avaliados por intermédio do alfa de Crombach, também obtiveram os índices de fidedignidade satisfatórios, sendo eles: Relações Sociais, alfa $=0,86$ (12 itens); Prestígio, alfa $=0,86$ (11 itens); Realização, alfa =0,95 (15 itens); e Estabilidade, alfa $=0,87$ (sete itens). Esses índices são indicativos de boa consistência interna da escala (qualidade e coerência) para medir a priorização e a satisfação dos valores relativos ao trabalho (Pasquali, 1999).

\section{Procedimento}

Após ser aprovada pelo CONEP e obter a permissão da Diretoria dos respectivos departamentos da Universidade onde se levou a cabo a pesquisa, os estudantes foram convidados a fazer parte do estudo. Realizava-se aos participantes a leitura do Termo de Consentimento Livre e Esclarecido e, após a assinatura do termo, era-lhes assegurado o sigilo no tratamento dos dados, assim como a possibilidade de retirada do consentimento a qualquer etapa do processo de pesquisa. $\mathrm{O}$ protocolo era preenchido individualmente pelo próprio participante, e as aplicações foram coletivas e em grupos de alunos na própria sala de aula deles. Toda a aplicação foi acompanhada por uma das pesquisadoras.

\section{Análise de dados}

Os dados coletados foram analisados por aglomerado e receberam tratamento estatístico, por meio do pacote estatístico SPSS para Windows, versão 11.0. Como as pontuações obedeciam a uma distribuição normal, foi utilizada a técnica de Análise de Variância ANOVA para a comparação de médias. Foram consideradas significativas as diferenças de médias estabelecidas em um nível de $p \leq 0,05$.

\section{Resultados e Discussão}

A análise das respostas identificou os dados apresentados na Tabela 1 .

Tabela 1

Comparação de média obtida na priorização dos valores relativos ao trabalho e na satisfação desses valores no ambiente laboral por homens e mulheres

\begin{tabular}{|c|c|c|c|c|}
\hline \multirow{2}{*}{ 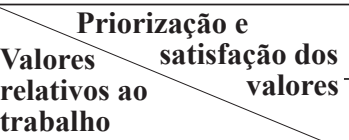 } & \multicolumn{2}{|c|}{ Média de priorização dos valores (VRT) } & \multicolumn{2}{|c|}{ Média de satisfação dos valores (SVRT) } \\
\hline & Homens & Mulheres & Homens & Mulheres \\
\hline Realização & $4,26 * a$ & $4,41 * b$ & 3,53 & 3,64 \\
\hline Relações sociais & 4,07 & 3,94 & 3,20 & 3,14 \\
\hline Prestígio & 3,02 & 2,99 & 2,76 & 2,72 \\
\hline Estabilidade & $3,99 * * a$ & $4,25 * * \mathrm{~b}$ & 2,95 & 2,81 \\
\hline
\end{tabular}

Legenda: * $p<0,05 ; * * p<0,01$

Os resultados da ANOVA indicam uma diferença significativa na priorização dada pelos homens e pelas mulheres em relação aos valores de estabilidade e realização. Desse modo, as mulheres tendem a buscar, no trabalho, prazer e realização profissional, independência de pensamento e ação, por meio da autonomia intelectual e da criatividade. Na mesma direção, as mulheres priorizam mais que os homens segurança e ordem na vida, na busca de suprir necessidades materiais. Não foram identificadas diferenças significativas quanto à satisfação ou realização dos valores laborais.

Os resultados demonstram haver diferenças estatisticamente significativas nas prioridades de valores relativos ao trabalho em relação a homens e mulheres. Essa análise indica uma tendência de as mulheres priorizarem valores relativos à realização e à estabilidade, ao passo que os homens tendem a considerar mais importantes, os valores de relações sociais e de prestígio como autoridade, sucesso profissional, poder de influência e relações sociais positivas.

Vale ressaltar que, a generalização desses dados acerca da diferença de gênero em relação aos valores relativos ao trabalho requer uma replicação do estudo, utilizando-se uma amostra que contemple um maior número de participantes, com diferentes níveis de escolaridade, faixa salarial, profissional, extensivo ao que representaria a população geral.

Cabe salientar que os dados deste artigo contradizem as priorizações femininas de valores universalistas relacionados à autotranscendência e coincidem com as priorizações masculinas de realização e prestígio identificadas por Schwartz e Rubel (2005). Em contrapartida, esses dados confirmam parcialmente os obtidos por Tamayo (1994) no que se refere à prioridade atribuída pelas mulheres aos valores de estabilidade. 
Tais diferenças poderiam estar relacionadas ao uso de escalas distintas para o estudo de valores gerais e laborais, porém a equivalência já identificada entre as duas escalas enfraquece tal hipotese (Porto, 2004). Outra hipótese explicativa para esse resultado poderia estar relacionada à influência da socialização no contexto laboral (e neste sentido há de se ressaltar as especificidades culturais de distintos contextos) sobre a formação dos valores relativos ao trabalho.

Há que se considerar também a possibilidade de busca de realização no trabalho como mecanismo compensatório das metas almejadas em outros contextos da vida, conforme assinala Porto (2004). Afinal, as diferenças de gênero permeiam todos os papéis desempenhados por homens e mulheres e, historicamente, de maneira menos favorável para as mulheres, o que torna compreensível que elas busquem diversas compensações no âmbito do trabalho.

As tendências de priorização pelas mulheres dos valores referentes à realização e pelos homens dos valores relacionados a prestígio coincidem com os resultados identificados por Porto (2004) em um grupo amostral diferente deste estudo.

Em contrapartida, a tendência feminina para priorizar estabilidade pode estar relacionada ao seu crescente papel de provedora familiar, e não à argumentação da teoria evolutiva, endossada por Schwartz e Rubel (2005), de fragilidade feminina. Considerando que $95 \%$ das mulheres brasileiras que trabalham mantêm uma jornada laboral dupla, executando ou chefiando atividades domésticas, depois de oito horas diárias de trabalho remunerado, parece ser incoerente pensar em fragilidade deste gênero. Percebe-se assim que, pelo menos em parte, a desigualdade entre gênero ao invés de diminuir se acentua, pois além de cumprir com as obrigações que lhes são historicamente atribuídas, como o cuidado da casa e da família, as mulheres estão assumindo de fato e de direito as responsabilidades relacionadas à manutenção da família.

Os valores laborais referentes à realização estão associados à busca de independência de pensamento e ação por meio da autonomia intelectual e da criatividade. Schwartz (2005, p. 25) associa esse valor à ênfase "na demonstração de competência, em termos de padrões culturais prevalentes e, portanto, aprovados socialmente". Considerando tal afirmação, parece oportuno observar que a mulher talvez esteja "andando em círculo", ou seja, mesmo que tenha logrado ingressar no mercado de trabalho buscando sua independência e autonomia, ainda espera, também no âmbito do trabalho, um reconhecimento de sua competência e aprovação social, demonstrado nos dados em que prioriza o valor realização. Nesse sentido, podemos dizer que se confirma a afirmação de Scott (1995, p. 75) de que o "mundo das mulheres faz parte do mundo dos homens e é criado nesse e por esse mundo masculino". Isto talvez explique em parte porque a maioria das mulheres ainda se mantém distante das posições de liderança nas organizações.

As diferentes priorizações dadas aos valores laborais por homens e mulheres poderiam estar sendo mantidas por intermédio de um dinâmico processo, cada vez mais estimulado pela maior participação da mulher no mercado de trabalho. Reportando ao modelo de congruência de crenças proposto por Rokeach (1973), a realização, por parte das mulheres, de tarefas condizentes com valores priorizados pelos homens, poderia estar acirrando estereótipos e emoções que subsidiariam atitudes de não aceitação da atuação feminina em áreas específicas como, por exemplo, as gerenciais ou aquelas que não impliquem em função de cuidadora.

A constatação de que homens e mulheres priorizam valores que, segundo a teoria de Schwartz, seriam conflitantes conduz à ideia de uma caracterização muito específica deles no complexo mundo do trabalho: a convivência com princípios e metas motivacionais absolutamente paradoxais. Remete ainda, à complexidade das questões de gênero, em que a mulher ainda perpetua o dilema entre a submissão e a autonomia. Além disso, também nos possibilita hipotetizar que a ideia da contraposição ao modelo feminino socialmente estabelecido, conforme descrito anteriormente, passe, na visão das mulheres, a assumir uma postura masculinizada em âmbitos da vida em que essa postura possa ser mais aceita e valorizada, como é o caso do contexto de trabalho.

Contribui para essa discussão o fato de não ter-se encontrado diferenças significativas na satisfação das prioridades axiológicas dos valores para homens e mulheres. Assim, pode-se postular que, embora existam diferenças entre gênero na priorização de valores - tipo de cargo assumido, por exemplo -, uma vez assumidos esses papéis, a repercussão na saúde mental e o atendimento das necessidades dos trabalhadores não são afetados pela variável gênero, e sim por outras variáveis tais como tempo no emprego e tipo de jornada.

\section{Considerações finais}

Considerando que os valores permeiam todos os papéis desempenhados por homens e mulheres em diversos contextos, as conclusões preliminares, aqui formuladas, adquirem especial importância para possíveis intervenções que objetivem a redução do sexismo no âmbito do trabalho. É fundamental oportunizar reflexões junto aos personagens do mundo do trabalho sobre os diversos fatores com os quais as representações do masculino e feminino estabelecem interface, extrapolando, ou melhor, não se restringindo apenas ao contexto organizacional imediato.

À guisa de conclusão, considerando a pouca quantidade de estudos referentes a gênero e valores, no âmbito da psicologia organizacional e do trabalho, é oportuno recomendar a realização de novas pesquisas sobre o tema, buscando identificar possíveis relações entre priorização axiológica laboral e socialização no trabalho, as relações familiares, a satisfação de mulheres e homens com o papel profissional que desempenham, diferenças geográficas regionais, trabalho doméstico versus trabalho remunerado e segmentação profissional. 


\section{Referências}

Abramo, L. (2002). A situação da mulher latino-americana. In D. G. Delgado, P. Capellin, \& V. Soares (Orgs.), Mulher e trabalho: Experiências de ação afirmativa. São Paulo: Boitempo.

Belo, R. P., Gouveia, V. V., Raymundo, J. S., \& Marques, C. M. C. (2005). Correlatos valorativos do sexismo ambivalente. Psicologia, Reflexão e Crítica, 18, 7-15.

Beutel, A. M., \& Marini, M. M. (1995). Gender and values. American Sociological Review, 60, 436-449.

Cheung, C. K., \& Scherling, S. A. (1999). Job satisfaction, work values, and sex differences in Taiwan 's organizations. The Journal of Psychology, 133, 563-575.

Ferreira, M. C., \& Assmar, E. M. L. (2005). Valores e sexismo no contexto do trabalho. In A. Tamayo \& J. B. Porto, Valores e comportamentos nas organizações (pp. 280-302). Petrópolis: Vozes.

Fischer, I. R. (2001). Gênero e exclusão social. Fundação Joaquim Nabuco (Trabalhos para discussão, no 113). Recuperado em 18 maio 2006, de http:// www.fundaj.gov.br

Fiúza, A. L. C. (2001). Mulheres nas políticas de desenvolvimento sustentável. In C. Bruschini, \& C. R. Pinto (Orgs.), Tempos e lugares de gênero (pp. 87-118). São Paulo: FCC/Editora.

Formiga, N. S. (2007). Valores humanos e sexismo ambivalente. Revista do Departamento de Psicologia UFF, 19, 381-396.

Instituto Brasileiro de Geografia e Estatística. (2006). Síntese de indicadores sociais. Recuperado em 12/06/2006, de http://www.ibge.gov.br 2006

Jensen, T. D., White, D. D., \& Singh, R. (1990). Impact of gender, hierarchial position, and leadership styles on work-related values. Journal of Business Research, 20, 145-152.

Manhardt, P. J. (1972). Job orientation of male and female college graduates in business. Personnel Psychology, 25, 361-368.

Mottaz, C. (1986). Gender differences in work satisfaction, work-related rewards and values, and the determinants of work satisfaction. Human Relations, 39, 359-378.

Pasquali, L. (1999). Instrumentos psicológicos: Manual prático de elaboração. Brasília: LabPAM / IBAPP.

Palermo, J., \& Evans, A. (2007). Relationschips between personal values and reported behavior on ethical scenarios for law students. Behavioral Sciences and Law, 25, 121-136.

Porto, J. B. (2004). Estrutura e transmissão dos valores laborais: Um estudo com estudantes universitários. Tese de Doutorado não-publicada, Universidade de Brasília, Brasília.

Porto, J. B., \& Tamayo, A. (2003). Escala de valores relativos ao trabalho: EVT. Psicologia: Teoria e Pesquisa, 19, 145-152.
Rokeach, M. (1973). The nature of human values. New York: Free Press.

Ros, M., Schwartz, H. S., \& Surkiss, S. (1999). Basic individual values, work values, and the meaning of work. Applied Psychology: An International Review, 48, 49-71.

Sagie, A., Elizur, D., \& Koslowsky, M. (1996). Work values: A theoritical overview and a model of their effects. Journal of Organizational Behavior, 17, 503-514.

Scott, J. (1995). Gênero: Uma categoria útil de análise histórica. Educação e realidade, 20(2), 71-99.

Schwartz, S. H. (1992). Universals in the content and structure of values: Theoretical advances and empirical tests in 20 countries. In M. Zanna, Advances in experimental social psychology (pp. 1- 65). Orlando: Academic.

Schwartz, H. S. (2005) Valores humanos básicos: Seu contexto e estrutura intercultural. In A. Tamayo, \& J. B. Porto(Orgs.), Valores e comportamento nas organizações. Petrópolis: Vozes.

Schwartz, H. S., \& Rubel, T. (2005). Sex differences in value priorities: Cross-cultural and multimethod studies. Journal of personality and social psychology, 89, 1010 - 1028.

Tamayo, A., \& Schwartz, H. S. (1993). Estrutura motivacional dos valores humanos. Psicologia: Teoria e Pesquisa, 9, 329-348.

Tamayo, A. (1994). Hierarquia dos valores transculturais brasileiros. Psicologia: Teoria e Pesquisa, 10, 269. 285.

Torres, M. A. S. (2006). A divisão sexual do trabalho: A inserção da mulher no mundo do trabalho. Trabalho apresentado no $1^{\circ}$ Seminário Nacional de Trabalho e Gênero, Goiânia.

Venturi, G., \& Recamán, M. (2005). As mulheres brasileiras no início do século XXI (Relatório dos direitos humanos no Brasil). Recuperado em 19 junho 2006, de http:// www2.fpa.org.br

Margareth Ribeiro Machado Santos e Silva é professora da Faculdade Estácio de Sá de Goiás.

Helenides Mendonça é Professora Titular da Faculdade de Psicologia da Pontifícia Universidade Católica de Goiás.

Daniela Sacramento Zanini é Professora Adjunto I da Faculdade de Psicologia da Pontifícia Universidade Católica de Goiás.

Recebido: 04/06/2008

$1^{a}$ revisão: 16/03/2009

$2^{a}$ revisão: $16 / 05 / 2009$

Aceite final: 11/06/2009 